\title{
Anal Stenosis
}

National Cancer Institute

\section{Source}

National Cancer Institute. Anal Stenosis. NCI Thesaurus. Code C78173.

Narrowing of the anal canal. 